\title{
RF/Microwave Discharge Plasma for Mercury-Free Lighting
}

\author{
Ahmad Nazri DAGANG*,**, Hideki MOTOMURA** and Masafumi JINNO** \\ *School of Ocean Engineering, Universiti Malaysia Terengganu, 21030 Kuala Terengganu, Malaysia \\ **Department of Electrical and Electronic Engineering, Ehime University, 3 Bukyo-cho, Matsuyama, Ehime 790-8577, Japan
}

Received December 15, 2013, Accepted May 26, 2014

\begin{abstract}
This research focused on the development of a mercury-free light source through the use of radio frequency (RF) and microwave discharges. RF at $13.56 \mathrm{MHz}$ and microwave at $2.45 \mathrm{GHz}$ were applied to generate the plasma. With the use of this kind of high frequency power generator, plasma can be ignited without using the electrodes inside the chamber or discharge tube, which could significantly increase the lamp lifetime. The potential for high luminance, excellent colour rendering and environmental-friendliness makes xenon the most promising candidate as the replacement for the mercury fluorescent lamp. In this research, RF energy at $13.56 \mathrm{MHz}$ was induced to the cylindrical discharge tube in order to investigate the characteristics of the xenon RF-inductively coupled plasma discharge as a light source. The luminance, spectral distribution and colour rendering index were measured. The values of the luminance and colour rendering index were comparative to the values for a conventional discharge lamp. For microwave plasma, in order to develop a planar-type lamp, surface wave plasma was applied. A few types (transverse, inclined, longitudinal) of slot antennas were tested and the lamp brightness and discharge uniformity were evaluated. The chamber window (front or rear) was coated with a three-colour phosphor from the combination of red, green and blue phosphors and the excitation effect was investigated. From phosphor coating and slot structure optimisations, lamp luminance and discharge uniformity could be improved.
\end{abstract}

KEYWORDS: inductively coupled plasma, surface wave plasma, mercury-free, electrode-less discharge, xenon

\section{Introduction}

Due to a growing concern about the environmental issues related to the disposal of toxic mercury found in the most lamp products, there have been intensified research efforts into developing mercury-free lamps. One of the promising candidates as an alternative to mercury is inert gas, that is, xenon, due to its non-toxicity, chemically stable and temperature-independence. The discharge method used in this research is an electrode-less discharge method which has already became one of the most important plasma producing technologies. Without an electrode, the lamps will be free from problems such as the internal chemical reaction and dark spots (due to electrode sputtering), more importantly, significant energy saving will be achieved as the lamp lifetime will be extended. An electrode-less discharge is a discharge that has no internal electrodes. It was discovered by Hittorf in 1884 and more complete observations were made soon after by Thomson and Tesla ${ }^{12)}$. Rapid developments in semiconductor technology and changes in the standards for electromagnetic interference (EMI) have been important steps in the exploitation of electrode-less discharges for lighting purposes.
There has been an active period of research on electrode-less discharges inside and outside of the lighting industry and much of the basic physics and technology is now well understood. With the absence of electrodes in generating and accelerating free electrons to ionise the gas, the electrode-less discharge needs an electromagnetic power at high frequency in order to generate the plasma. Electrode-less discharges can be divided into three groups, namely, capacitively coupled plasma (CCP), inductively coupled plasma (ICP), and microwave plasma. Electrode-less discharge lamps are not limited to lighting purposes, for example, research on their application as wireless communication tools is ongoing ${ }^{3}$. Research on electrode-less mercury-free lamps is also not limited to inert gas. For example, the microwave driven indium-iodide-based discharge lamp with high colour rendering has been proposed ${ }^{4}$. Research on electrode-less discharge for lighting applications has been undertaken by many researchers for decades. Some lamp producers have already commercialised mercurybased electrode-less lamp products ${ }^{5}$. However, as far as the mercury-free electrode-less lamp is concerned, no outstanding results have been reported. There was an 
active period of research on mercury-free electrode-less lamps using mainly microwave plasma during the late 1990s and early 2000s but since then the interest has declined due to the rapid development of solid state lighting. For instance, Shido et al. ${ }^{6}$ ) used additives such as $\mathrm{NaI}$ in high pressure argon using microwave discharge, and Dolan et al. ${ }^{7)}$ used a high pressure sulfur microwave discharge. Most of the previous research on mercury-free electrode-less discharge used high pressure gas with the aim to develop an alternative for the high intensity discharge (HID) lamp, but the outcome could not compete with the current product in terms of the lumens output and lamp efficiency. The work presented here is focused more on the domestic lighting application using low pressure xenon, with two types of discharge methods in order to provide an alternative for different purposes.

The research only deals with xenon as an alternative element. In general, at low pressure discharge, xenon emits strong resonance and excimer emissions in vacuum ultra-violet (VUV) regions at $147 \mathrm{~nm}$ and $172 \mathrm{~nm}$, and at high pressure discharge it is able to emit strong continuum emissions in the visible regions. The UV lines from xenon are the nearest to the lines that can be obtained from mercury at $254 \mathrm{~nm}$ and this is important in order to have good efficiency in converting UV to visible light through phosphor in the fluorescent lamp. The continuum emission can produce an intense white light, which is primarily used as a vehicle headlight. However, the work presented here is concentrated on low pressure discharge with the aim to obtain strong continuum emissions from ICP and good UV for the visible conversion of phosphor from surface wave plasma (SWP).

This research investigates the development of an electrode-less discharge for domestic light application as a mercury-free source. Two types of electrode-less discharges, namely, RF-ICP plasma for the cylindricaltype lamp and microwave plasma for the planar-type lamp, are studied. An ICP is a type of plasma in which the energy is supplied by electrical currents which are produced by electromagnetic induction by time-varying magnetic fields. When a time-varying electric current is passed through the coil, it creates a time-varying magnetic field around it, which in turn induces azimuthal electric currents that ionise and produce plasma. The type of microwave plasma applied in this study is SWP. SWP is a microwave electrode-less discharge where the sustaining electric field is provided by a wave that creates its own propagating medium. There is no magnetic coil in SWP, so a compact plasma source can be obtained and no internal electrode is needed, making it free from the problem of impurity contamination due to electrodes. By using SWP with a slot antenna, large-area plasma can be obtained ${ }^{8)}$ which it is believed, can be applied to produce high luminous light sources. Exploit- ing the aforementioned advantages of these two types of electrode-less discharges, the work presented here aimed to provide an experimental-based fundamental guide to the development of a light source in terms of luminance and colour output. This could be an important guideline for further investigation into the use of $\mathrm{RF}$ and microwave plasmas as a mercury-free electrode-less light source.

\section{Experimental setup 2.1 RF plasma}

ICP discharge was induced to the lamp by coupling the $\mathrm{RF}$ energy at $13.56 \mathrm{MHz}$ through a capacitive matching network. The RF current flowing in the coil generated an RF electric field, accelerated the free electron, caused ionisation and produced the plasma. The discharge was maintained by the time-varying magnetic field created by the primary induction coil placed outside the plasma.

A diagram of the setup for the luminance and spectrum measurement is shown in Figure 1. The lamp was $200 \mathrm{~mm}$ in length and the inner and outer diameters were $26 \mathrm{~mm}$ and $30 \mathrm{~mm}$, respectively. The glass material was borosilicate (Pyrex) and 4 turns of copper wire with a diameter of $1.6 \mathrm{~mm}$ and $10 \mathrm{~mm}$ pitch were set in the middle section of the discharge tube. The xenon gas pressure changed from 0.1 Torr to 50 Torr at room temperature. The input power was adjusted from 20 to $100 \mathrm{~W}$. The RF generator used was a Cesar $1310 \mathrm{RF}$ Generator, and the luminance was measured by the Topcon BM-7 luminance calorimeter. The emission spectra were measured by the USB spectrometer Ocean Optics USB-2000 and recorded to a personal computer. Its spectroscopic sensitivity was calibrated using the Bentham SRS8-Q standard light sources.

Colour rendering was measured using calibrated spectrum data. The property of a light source is quantitatively represented as the colour rendering index (CRI). The general CRI, $R_{\mathrm{a}}$ of a certain light source is calculated by comparing its spectral distribution with that of the standard light source. Higher $R_{\mathrm{a}}$ means bet-

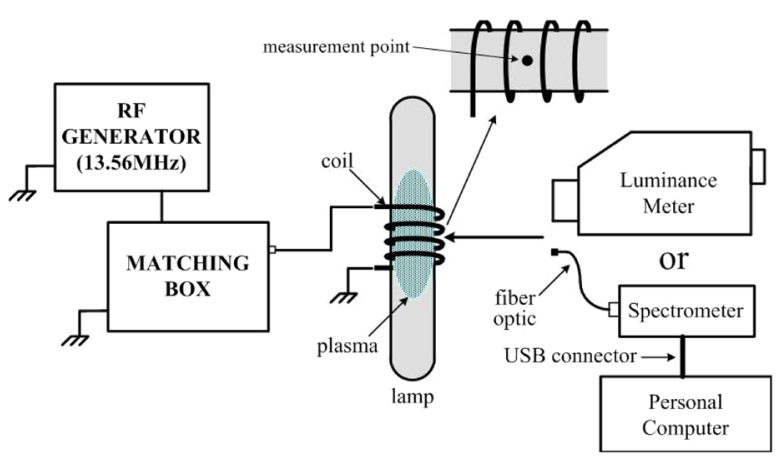

Figure 1 Connection diagram for luminance and spectrum measurements 


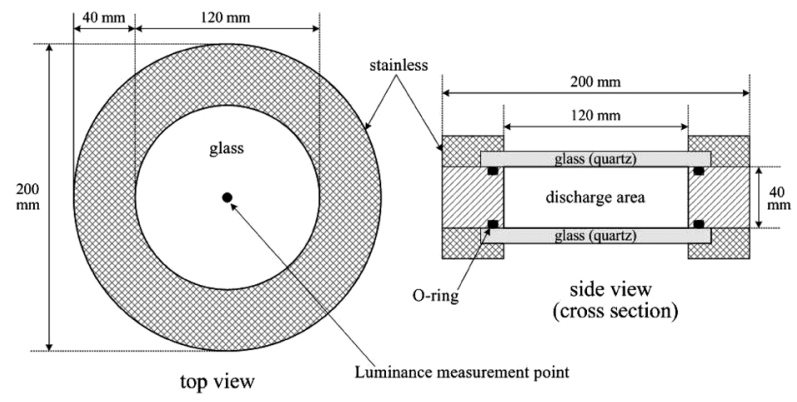

Figure 2 Diagram of chamber used in the experiment

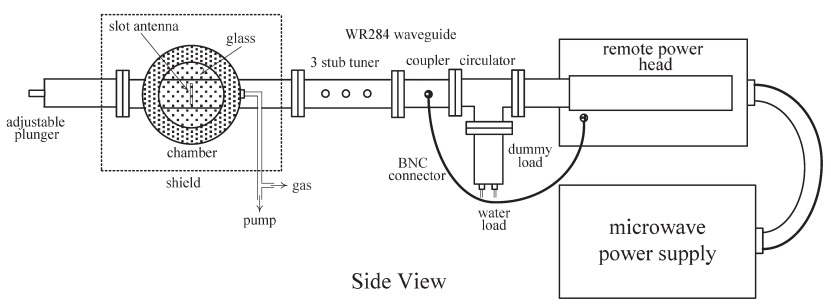

Figure 3 Setup diagram for surface wave plasma discharge

ter colour rendering properties up to a maximum value of 100. In the present study, the $R_{\mathrm{a}}$ was derived using the Bentham BenWin+colour rendering program.

\subsection{Microwave plasma}

A $2.45 \mathrm{GHz}$ microwave generator with maximum power up to $1.5 \mathrm{~kW}$ (Astex S-1500) was used. This power source is designed for use with a separate power head. The power head includes a magnetron, overload sensor, forced air cooling and waveguide output. A BNC connector is used to connect the power head, providing an interface to a reflected power detector on a separately supplied directional coupler. The microwave power supplied is terminated with an adjustable plunger at the end of the system. A diagram of the system is shown in Figure 3. In the study, a microwave frequency of $2.45 \mathrm{GHz}$ was fed into the discharge chamber through a slot antenna contacting the windows. The windows (front and rear) were made of quartz (fused silica) with a size of $12 \mathrm{~cm}$ in diameter. A diagram of the chamber used in the experiment is shown in Figure 2. The chamber was covered by aluminum mesh for shielding purposes in order to avoid microwave interference with the surrounding instruments. It was vacuumed using rotary and turbo molecular pumps up to $10^{-3}$ Torr. Xenon gas was filled at a pressure range from 0.3 Torr to 10 Torr at room temperature. The input power was varied from 300 to $600 \mathrm{~W}$ and the reflected power was minimised to less than $20 \mathrm{~W}$ by adjusting the 3 -stub tuner and plunger. The dimensions of the rectangular waveguide and slot antenna were $72.1 \mathrm{~mm} \times 34 \mathrm{~mm}$ and $61 \mathrm{~mm} \times 6.1 \mathrm{~mm}$, respectively. Luminance was measured using the Topcon BM-7 luminance calorimeter placed

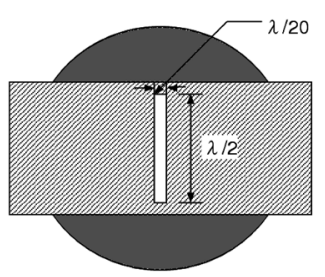

Slot A (transverse, $\left.90^{\circ}\right)$

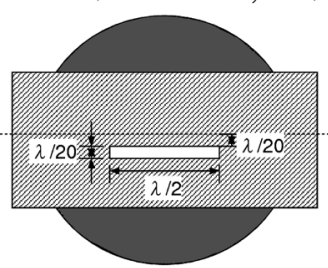

Slot C (longitudinal, $0^{\circ}$ )

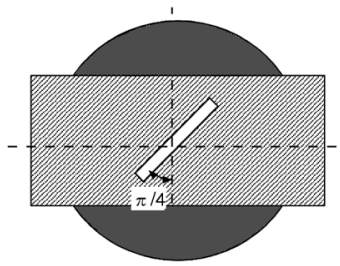

Slot B (inclined, $45^{\circ}$ )

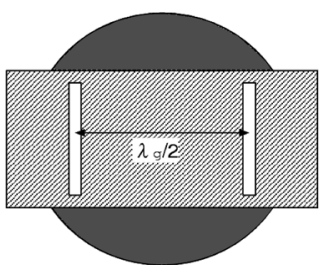

Slot D (transverse, 2 slots)
Figure 4 Slot antenna structures

$50 \mathrm{~cm}$ apart from the measurement point. The measurement point was set at the centre of the glass chamber, as shown in Figure 2. The structures of the slot antenna are shown in Figure 4 with transverse, inclined $\left(45^{\circ}\right)$ and longitudinal types. They were calculated based on the value of wavelengths in free space $\lambda(122 \mathrm{~mm})$ and in waveguide $\lambda_{\mathrm{g}}(144 \mathrm{~mm})$. In order to evaluate the effect of the phosphor, the glass windows were coated on either the front or rear window using the mixture of red, green and blue phosphors in the same ratio in order to produce white light. Their luminance was measured for both conditions. In order to evaluate the discharge uniformity numerically, the discharge image was taken using a digital camera and evaluated through image processing using Matlab programming.

\section{Results and discussion}

\subsection{Inductively coupled plasma}

\subsubsection{Relation between luminance, luminous flux and gas pressure}

The relation between luminance and the xenon gas pressure at $50 \mathrm{~W}$ and $100 \mathrm{~W}$ of input power is shown in Figure 5. When the pressure decreased from 50 to 1 Torr, the luminance also decreased. At high pressure, electron collision frequency is high due to the high molecular densities of gases which cause more electron collisions. Luminance again increased when the gas pressure decreased from 1 to 0.2 Torr. In RF discharge, there are two types of heating mechanisms, namely, ohmic heating (collisional heating) and stochastic heating (collisionless heating). Theoretically, when $v_{\mathrm{m}} \gg \omega$, collisional heating is dominant, and when $v_{\mathrm{m}} \ll \omega$, collisionless heating is dominant ${ }^{9}$ ) (where, $v_{\mathrm{m}}$ is the electron-neutral momentum transfer frequency and $\omega$ is the power supply frequency). From the approximate calculations, the boundary condition was assumed to be $v_{\mathrm{m}}=\omega$, the xenon pressure range was from $4.5 \mathrm{mTorr}$ to 
0.31 Torr at electron temperature, $\mathrm{Te}=1$ to $6.3 \mathrm{eV}$. From the results, it is deduced that the effect of stochastic heating started to appear when the xenon pressure was less than 1 Torr. Godyak et al. ${ }^{10)}$ reported that at ICP argon discharge, when the gas pressure is decreased, the ratio of stochastic heating to ohmic heating increased in which the electron density decreases and the electron temperature increases. Therefore, the luminance behaviour as shown in Figure 5 is considered to be due to the different heating mechanism in ICP discharge that gives high luminance at below and above 1 Torr. A detailed calculation is shown in the appendix. On the other hand, based on the luminance values that obtained, luminous flux $(F)$ can be calculated using ${ }^{11)}$,

$$
F=2 \Pi^{2} r h L
$$

where $r$ is the lamp inner radius, $h$ is the plasma length and $L$ is the luminance. In an approximate calculation, $r$ is $0.013 \mathrm{~m}, h$ is on average $0.05 \mathrm{~m}$ (plasma produced along the coil) and $L$ is assumed to be the same value from end to end of the plasma column. Table 1 shows a few selected pressures and their luminous flux values. In terms of the lamp efficacy (lumens per watt), the lamp is still far from the value of commercial lamps and one of the reasons is the energy loss at the coil. Nevertheless, there are still many parameters such as the coil thickness, turns and pitch that can be changed in order to improve the efficacy and reduce the heat loss.

\subsubsection{Color rendering and spectrum characteristics}

Figure 6 shows the relation between the CRI and gas pressure at xenon ICP $100 \mathrm{~W}$. Similar to the luminance characteristics, the CRI value also increased when the gas pressure increased at 1 to 50 Torr, where the CRI values ranged from 80 to 85 . The CRI decreased when

Table 1 Xenon pressures and calculated luminous flux

\begin{tabular}{|l|c|c|c|c|c|}
\hline Gas pressure (Torr) & 0.2 & 0.5 & 1 & 10 & 50 \\
\hline Luminous flux (lm) at $100 \mathrm{~W}$ & 142 & 96 & 24 & 95 & 99 \\
\hline
\end{tabular}

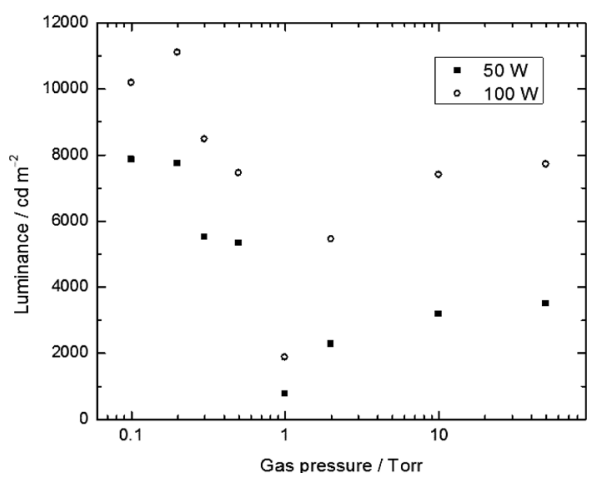

Figure 5 Relation between luminance and gas pressure at $50 \mathrm{~W}$ and $100 \mathrm{~W}$ the pressure was around 1 Torr and rose again when the gas pressure decreased from 1 to 0.2 Torr. CRI was the highest in the case of 0.2 Torr where the index value was $R_{\mathrm{a}}=95$. This is a high index value compared to the average CRI for a fluorescent lamp which is at 80 .

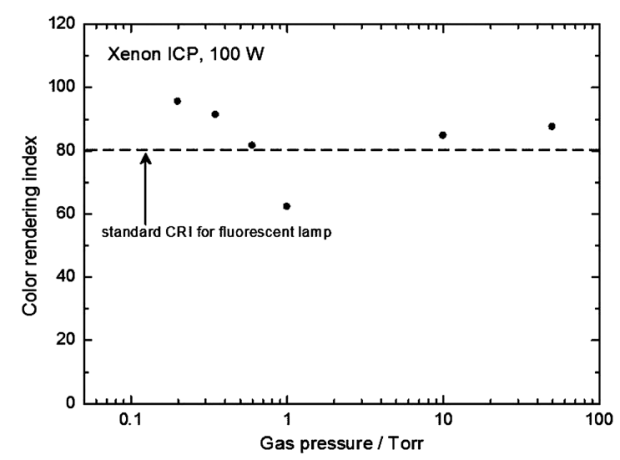

Figure 6 Relation between $\mathrm{CRI}$ value and gas pressure, xenon ICP at $100 \mathrm{~W}$

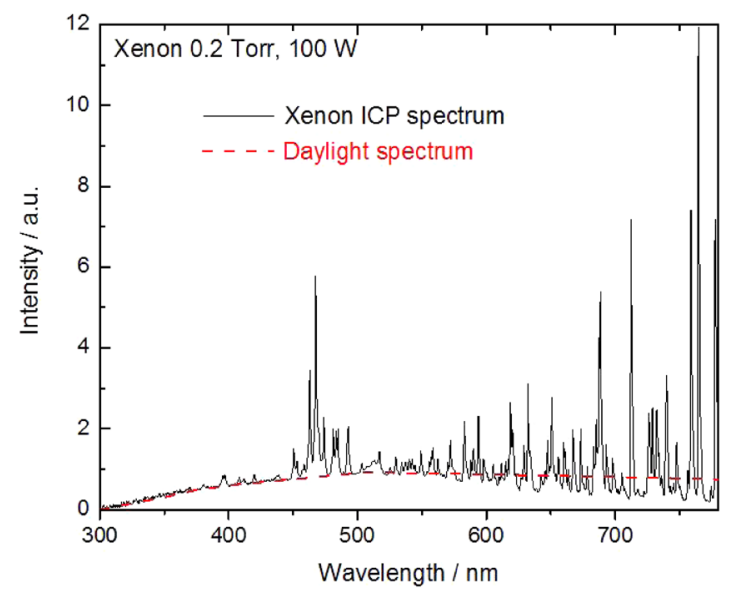

Figure 7 Relation between $\mathrm{CRI}$ value and gas pressure, wavelength at $300-780 \mathrm{~nm}$, xenon pressure at 0.2 Torr, input power at $100 \mathrm{~W}, R_{\mathrm{a}}=95$

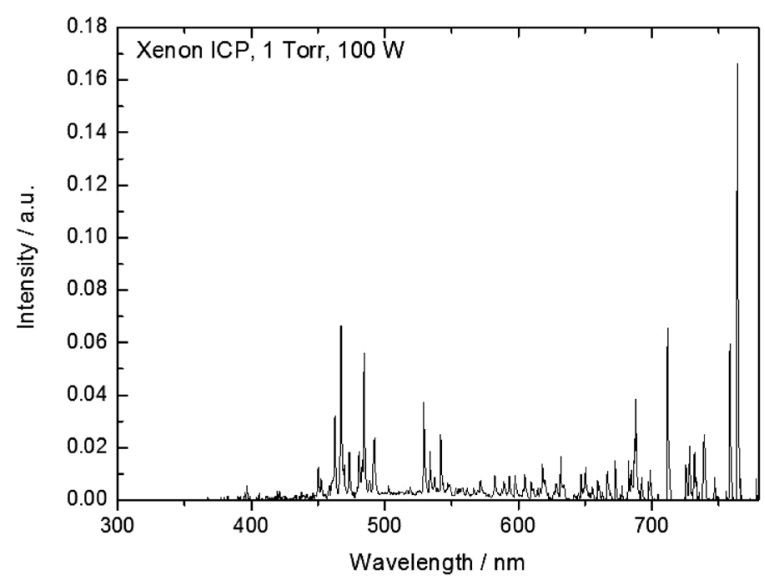

Figure 8 Relation between $\mathrm{CRI}$ value and gas pressure, wavelength at $300-780 \mathrm{~nm}$, xenon pressure at 1 Torr, input power at $100 \mathrm{~W}, R_{\mathrm{a}}=63$ 

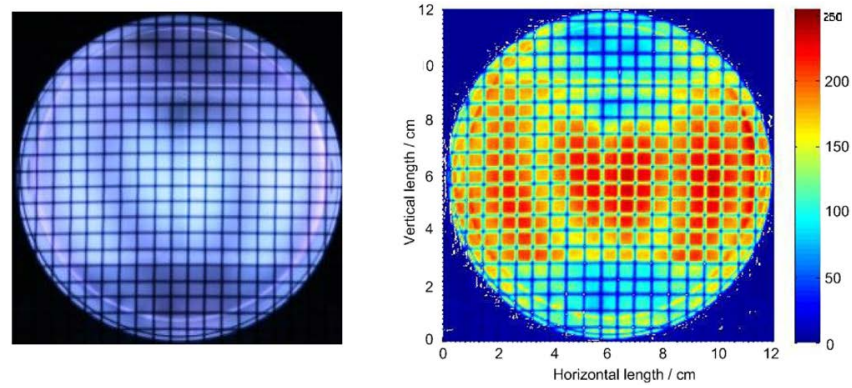

Figure 9 Discharge image and image processing for slot $A$
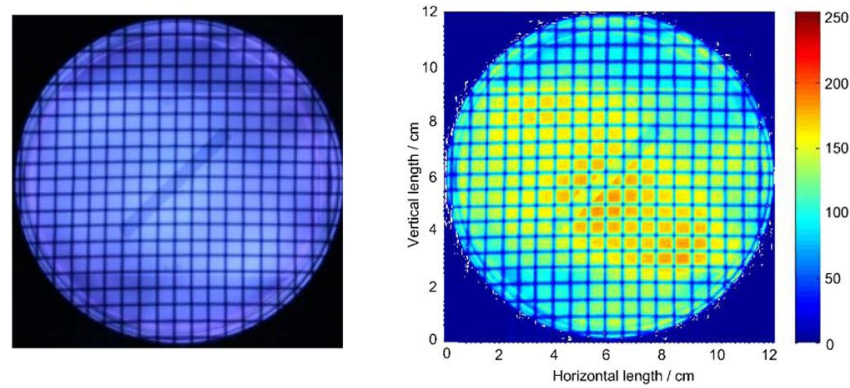

Figure 10 Discharge image and image processing for slot $B$

High CRI shows that the element of red, green, blue that can be obtained from xenon ICP at above condition is in good balance. The spectra of visible emission for each condition at 0.2 Torr and 1 Torr are shown in Figure 7 and Figure 8, respectively. As shown in Figure 7, the continuum visible emission was strong and lay on the entire visible area. On the other hand, the spectrum in Figure 8 shows that the continuum visible emission was weak where the line spectra were dominant. The high luminance and high CRI values of the ICP xenon lamp were related to this continuum visible emission which was either from the electron-ion recombination radiation or free-free transition of bremsstrahlung radiation $^{12)}$, or from both of these radiations. Good colour rendering means that the lamp performance was close to natural daylight and, from Figure 8, the continuum emission of the xenon ICP was similar to the daylight continuum spectrum up to around $650 \mathrm{~nm}$, giving the lamp a CRI value of 95 .

\subsection{Surface wave plasma}

\subsubsection{Effect of slot antenna structures}

The discharge images and image processing results are shown in Figure 9 to Figure 12 with four types of slot antenna structures. Table 2 presents the numerical evaluation of the slot antenna structures from image processing in terms of lamp intensity (brightness), standard deviation and light uniformity which is the ratio of deviation to brightness. Lamp brightness was calculated from all pixels taken from the images. From the discharge images as can be seen in the figures, a big difference can be seen in brightness especially for slots A
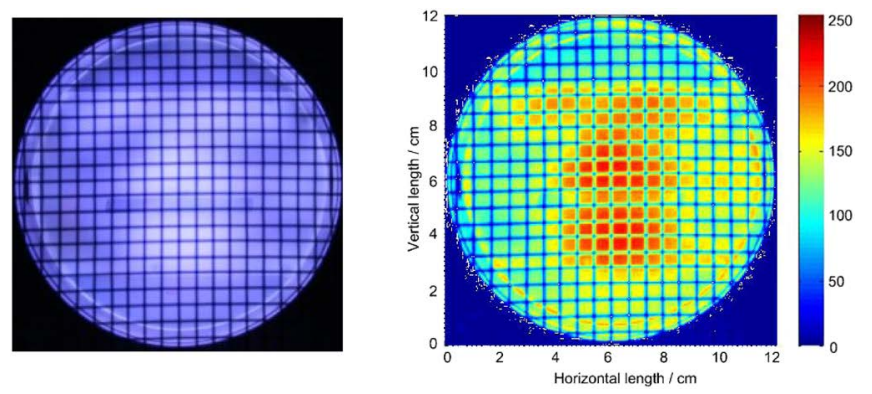

Figure 11 Discharge image and image processing for slot $\mathrm{C}$
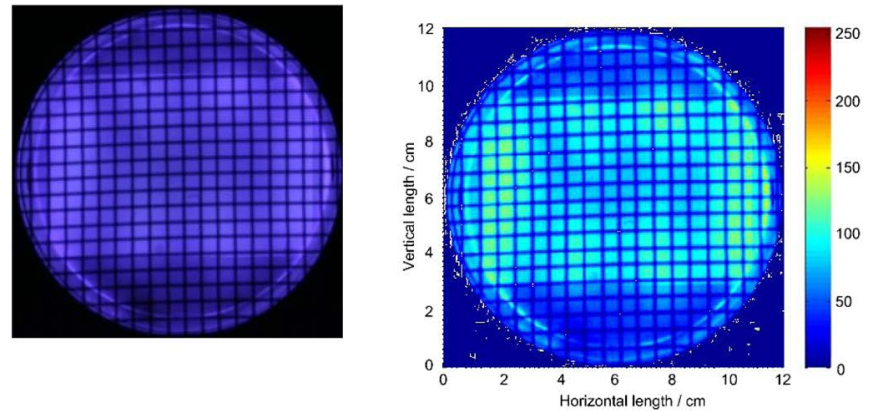

Figure 12 Discharge image and image processing for slot $D$

Table 2 Discharge brightness and uniformity (without phosphor)

\begin{tabular}{|c|c|c|c|}
\hline Xenon 1 Torr & $\begin{array}{c}\text { Intensity } \\
\text { (brightness) }\end{array}$ & $\begin{array}{c}\text { Standard } \\
\text { Deviation }\end{array}$ & $\begin{array}{c}\text { Uniformity } \\
\text { (deviation to } \\
\text { brightness) }\end{array}$ \\
\hline Slot A & 132 & 53 & 0.40 \\
\hline Slot B & 106 & 39 & 0.37 \\
\hline Slot C & 121 & 45 & 0.37 \\
\hline Slot D & 60 & 30 & 0.50 \\
\hline
\end{tabular}

and C compared to slots B and D. However, their discharge uniformity was poor. For discharge uniformity evaluation, slot $\mathrm{D}$ showed the lowest value of deviation meaning it was the most homogenous compared to the others. In terms of slot angle, the production of plasma was better at $90^{\circ}$ compared to $45^{\circ}$ and $0^{\circ}$. This could be due to the position of the surface wave (peak position) which was adjusted to the maximum level. In terms of the number of slots (slot D), plasma was poorly produced with two slots. This is because it was difficult to adjust the position of the surface wave to the peak position for both holes (slots). However, when the number of slots increased, there was an improvement in discharge uniformity.

\subsubsection{Luminance and effect of phosphor}

The relation between luminance and input power at 0.3 Torr when the input power was varied from 300 to $600 \mathrm{~W}$ is shown in Figure 13. Only a small luminance change was found when the input power changed. It is 


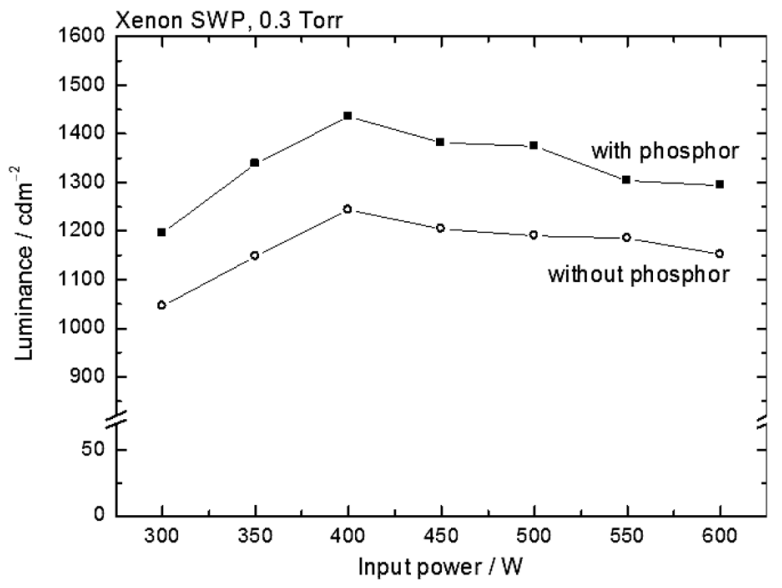

Figure 13 Relation between luminance and input power at 0.3 Torr, with and without phosphor (phosphor coated on front window)

thought that the input power was not absorbed efficiently and was transformed to heat loss. Figure 14 shows the relation between luminance and gas pressure when the gas pressure was varied from 0.3 to 10 Torr for both phosphor-coated and uncoated conditions. The luminance increased when the gas pressure decreased. It was found that an improvement of luminance was obtained when phosphor was used. As shown in Figure 13, at higher pressures of 5 Torr and 10 Torr, luminance was very low. However, the rate at which the luminance increased when using phosphor was high compared to the rate of increase at lower pressure. For instance, at an input power of $300 \mathrm{~W}$, the rate of luminance increase was $77 \%$ (65 to $115 \mathrm{~cd} / \mathrm{m}^{2}$ ) for 5 Torr and $50 \%\left(312\right.$ to $\left.468 \mathrm{~cd} / \mathrm{m}^{2}\right)$ for 1 Torr, whereas the rates were $15 \%$ at 0.3 Torr $\left(1045\right.$ to $\left.1200 \mathrm{~cd} / \mathrm{m}^{2}\right)$ and $9 \%\left(900\right.$ to $\left.982 \mathrm{~cd} / \mathrm{m}^{2}\right)$ at 0.5 Torr. Thus, the UV excitation of phosphor was more dominant in higher pressure conditions. Since this ratio of luminance increase may strongly depend on the phosphor thickness, it was expected that a higher luminance would be obtained by optimising the phosphor thickness. This condition was created when phosphor was coated on the front (from the observer) window. There was an improvement in luminance compared to the uncoated condition but the difference was not significant.

When phosphor was coated on the rear (slot antenna side) window, a significant increase of luminance was observed. As shown in the discharge image in Figure 15 , luminance increased more than five times compared to the front window coated chamber (from 982 to $5248 \mathrm{~cd} / \mathrm{m}^{2}$ ). This might have been due to the distance between the windows. Plasma was produced through the slot antenna which was directly attached to the rear window and the distance between the rear and front windows was $4 \mathrm{~cm}$. Because of this distance, the resonance line needed for phosphor excitation was ex-

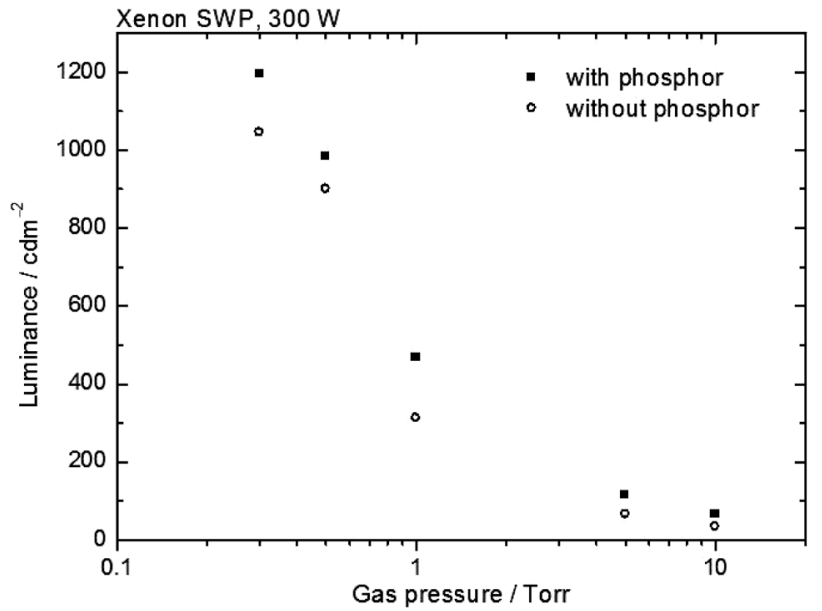

Figure 14 Relation between luminance and gas pressure at $300 \mathrm{~W}$, with and without phosphor (phosphor coated on front window)

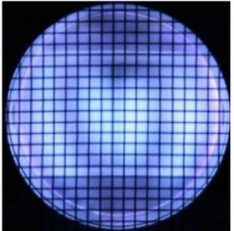

(a)

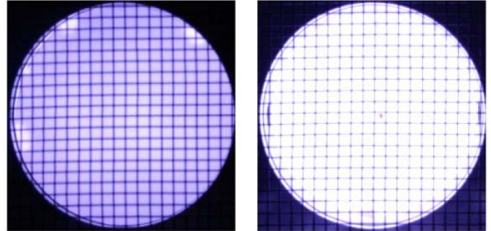

(b) (c)
Figure 15 Discharge images for three conditions, (a) without coating $900 \mathrm{~cd} \mathrm{~m}^{-2}$, (b) front coating, $982 \mathrm{~cd} \mathrm{~m}^{-2}$, (c) rear coating $5248 \mathrm{~cd} \mathrm{~m}^{-2}$ at 0.5 Torr

periencing self-absorption before it reached the phosphor at the front window. In contrast, the phosphor could be excited at the rear window soon after plasma was produced. However, considering the heat loss was higher on the antenna side, the phosphor might be broken due to this overheating condition.

\section{Conclusion}

The characteristics of cylindrical-type xenon ICP and planar-type xenon SWP lamps were investigated in this study. For the ICP lamp, the luminance and CRI values depended on the xenon gas pressure. Low-pressure xenon at 0.2 Torr delivered the best results, with higher luminance and CRI values. For the planar-type SWP lamp, in order to obtain high luminance with high efficacy, it appeared that the lower input power would be more effective due to high energy loss at the higher input power. Homogenous discharge was observed using the longitudinal slot and by coating a phosphor on the rear window, in this condition phosphor excitation improved, resulting in relatively high luminance up to $5000 \mathrm{~cd} / \mathrm{m}^{2}$. It is believed that optimisation of the xenon pressure (below 0.5 Torr) and phosphor coating condition (mixing ratio, thickness) can improve the luminance as well as the luminous flux. 


\section{Acknowledments}

The authors would like to thank technical staff member, Mr. Hisayoshi Kurokawa, for his assistance in lamp making and also postgraduate and undergraduate students, Mr. Kondo, Mr. Takeda and Mr. Sato, for their contributions to the experimental works. Part of this work was supported by Phoenix Electric Co., Ltd.

\section{References}

(1) Thompson, J. J.: On the discharge of electricity through exhauted tubes without electrodes, Philosophical Magazine, Vol. 32, pp. 321-336 (1891).

(2) Wharmby, D. O.: Electrodeless lamp for lighting: a review, IEE Proceedings-A, Vol. 140, pp. 465-473 (1993).

(3) Gilliard, R. P., Vincentis, M. D., Hafidi, A., O'Hare, D. and Hollingsworth, G.: Operation of the Li-Fi light emitting plasma in resonant cavity, IEEE Transaction on Plasma Science, Vol. 39, No. 4, pp. 1026-1033 (2011).

(4) Kaiser, C., Ogun, C. M., Kling, R. and Heering, W.: Electrodeless mercury-free microwave-driven indium-iodide-based high-intensity discharge with high color rendering index, IEEE Transaction on Plasma Science, Vol. 41, No. 1, pp. 77-81 (2013).

(5) Kido, H., Hiramatsu, K., Ogasawara, H. and Yamamoto, S.: High efficiency low-frequency electrodeless lamp system "Everlight", Matsushita Technical Report, pp. 10-15 (2005) (in Japanese).

(6) Shido, M., Nakamura, T., Serita, T. and Kando, M.: High-pressure microwave discharges in a compact quartz tube for an electrodeless high intensity discharge lamp, IEEJ Trans. Fundamentals and Materials, Vol. 125, No. 6, pp. 495-500 (2005).

(7) Dolan, J. T., Ury, M. G. and Wood, C. H.: Lamp Including Sulfur, US Patent, 4504076, pp. 1-9 (1995).

(8) Nagatsu, M., Morita, S., Ghanashev, I., Ito, A., Toyoda, N., Sugai, H.: Effect of slot antenna structures on production of large-area planar surface wave plasma xxcited at $2.45 \mathrm{GHz}$, J. Phys. D: Appl. Phys., Vol.. 33, pp. 1143-1149 (2000).

(9) Lieberman, M. A. and Lichtenberg, A. J.: Principles of Plasma Discharges and Materials Processing, 2nd ed., John Wiley \& Son (1994).

(10) Godyak, V. A., Piejak, R. B. and Alexandrovich, B. M.: Effective electron collision frequency and electrical conductivity of radio frequency plasma, J. Appl. Phy., Vol. 85, No. 6, pp. 3081-3083 (1999).

(11) Illuminating Institure of Japan: Illuminating Engineering, Ohm Publishing (1991)(in Japanese).

(12) Haraguchi, H.: Fundamental and application of ICP spectroscopy, Kodansha Scientific (1986) (in Japanese).

\section{Appendix}

The approximate calculation of gas pressure was done under the condition of electron-neutral collision frequency, $v \mathrm{~m}$, being equal to angular frequency $\omega$. The values used for electron mass $(m)$, Boltzmann constant $(k)$, collision cross-section area $\left(\sigma_{\mathrm{m}}\right)$ and angular frequency $(\omega)$ are as below while $P_{\mathrm{c}}{ }^{9)}$ is a unitless property of the collision probability. Below is the list of constant values that used.

$$
\begin{aligned}
& m=9.1095 \times 10^{-31} \mathrm{~kg} \\
& k=1.381 \times 10^{-23} \mathrm{JK}^{-1} \\
& \sigma_{\mathrm{m}=}=2.87 \times 10^{-21} P_{\mathrm{c}} \mathrm{m}^{2} \\
& \omega=8.52 \times 10^{7} \mathrm{~s}^{-1}
\end{aligned}
$$

The calculation was made at the highest and lowest xenon collision probability according to the figure of probability of collision in reference 9 . When $k T_{\mathrm{e}}=m v_{\mathrm{e}}^{2} / 2$, the velocity $v_{\mathrm{e}}$ is defined and the calculation is made under the condition of $T \mathrm{e}=1 \mathrm{eV}$ and $6.25 \mathrm{eV}$ (approximate value $)^{9}$. When $1 \mathrm{eV}=1.602 \times 10^{-19} \mathrm{~J}, v_{\mathrm{e}}$ is calculated as below.

$$
\begin{aligned}
v_{e} & =\sqrt{\frac{2 \mathrm{eV}}{m}} & & T_{\mathrm{e}}=1 \mathrm{eV} \text { (lowest) } \\
& =5.931 \times 10^{5} \mathrm{~ms}^{-1} & & \\
v_{e} & =\sqrt{\frac{2 \mathrm{eV}}{m}} & & T_{\mathrm{e}}=6.25 \mathrm{eV} \text { (highest) } \\
& =1.489 \times 10^{6} \mathrm{~ms}^{-1} & &
\end{aligned}
$$

With an approximate values $P_{\mathrm{c}}=5$ for $T_{\mathrm{e}}=1 \mathrm{eV}$, and $P_{\mathrm{c}}=140$ for $T_{\mathrm{e}}=6.25 \mathrm{eV}, \sigma_{\mathrm{m}}=1.435 \times 10^{-20}$ and $4.018 \times 10^{-19}$ were obtained respectively. Assuming the boundary condition as $v_{\mathrm{m}}=\omega$, the calculation can be done as follow.

$$
\begin{aligned}
v_{m} & =n\left\langle\sigma_{m} v_{e}\right\rangle \\
P & =n k T, n=\frac{P}{k T},(T=300 \mathrm{~K}) \\
\omega & =\frac{P}{k T}\left\langle\sigma_{m} v_{e}\right\rangle \\
P & =\frac{\omega k T}{\left\langle\sigma_{m} v_{e}\right\rangle}, T_{e}=1 \mathrm{eV} \\
& =0.311 \text { Torr } \\
P & =\frac{\omega k T}{\left\langle\sigma_{m} v_{e}\right\rangle}, T_{e}=6.25 \mathrm{eV} \\
& =0.0045 \text { Torr }
\end{aligned}
$$

Thus, at the boundary condition xenon gas pressured ranged from $4.5 \mathrm{~m}$ Torr to 0.3 Torr. 